\title{
Optic neuropathy after anterior communicating artery aneurysm clipping: 3 cases and techniques to address a correctable pitfall
}

\author{
Joseph R. Linzey, BS, ${ }^{1}$ Kevin S. Chen, MD, ${ }^{2}$ Luis Savastano, MD, ${ }^{2}$ B. Gregory Thompson, MD, ${ }^{2}$ and \\ Aditya S. Pandey, MD²
}

${ }^{1}$ University of Michigan Medical School, and 2Department of Neurosurgery, University of Michigan, Ann Arbor, Michigan

Brain shifts following microsurgical clip ligation of anterior communicating artery ( $A C D A)$ aneurysms can lead to mechanical compression of the optic nerve by the clip. Recognition of this condition and early repositioning of clips can lead to reversal of vision loss.

The authors identified 3 patients with an afferent pupillary defect following microsurgical clipping of ACoA aneurysms. Different treatment options were used for each patient. All patients underwent reexploration, and the aneurysm clips were repositioned to prevent clip-related compression of the optic nerve. Near-complete restoration of vision was achieved at the last clinic follow-up visit in all 3 patients.

Clip ligation of $\mathrm{ACOA}$ aneurysms has the potential to cause clip-related compression of the optic nerve. Postoperative visual examination is of utmost importance, and if any changes are discovered, reexploration should be considered as repositioning of the clips may lead to resolution of visual deterioration.

https://thejns.org/doi/abs/10.3171/2017.2.JNS162654

KEY WORDS afferent pupillary defect; anterior communicating artery aneurysm; intracranial aneurysm; optic nerve injuries; surgical clips; vascular disorders

A s many as $30 \%-37 \%$ of all cerebral aneurysms occur within the region of the anterior communicating artery (ACOA) ${ }^{1,4,6}$ Due to their proximity to the optic apparatus, ACoA aneurysms may present with symptoms of compressive optic neuropathy (CON). ${ }^{3,9}$ Multiple reports have described exacerbation of CON secondary to aneurysm expansion following coil embolization. . $7,8,10^{\mathrm{Mi}-}$ crosurgical clipping of ACoA aneurysms has been reported to result in favorable optic nerve function when compression by the aneurysm is suspected, as the aneurysm can be decompressed after clipping. ${ }^{2}$ However, clip-related CON after microsurgical treatment has not been well described. We report 3 cases in which an afferent pupillary defect (APD) was detected following clip ligation of ACoA aneurysms, in which reexploration with repositioning of the clip led to resolution of visual symptoms.

\section{Case Reports}

Case 1

A 61-year-old woman presented to the emergency department with severe headache and lethargy. At presentation she demonstrated a Hunt and Hess Grade V and World Federation of Neurosurgical Societies (WFNS) Grade V subarachnoid hemorrhage (SAH). CT demonstrated modified Fisher Grade 4 SAH (Fig. 1). A diagnostic cerebral angiogram revealed an ACoA aneurysm.

We performed a right-sided frontotemporal craniotomy for microsurgical clip ligation of the aneurysm., applying a Mizuho (Mizuho Medical Co.) MRI-compatible bayoneted clip applied to the aneurysm neck. Twenty-four hours following the procedure, there was concern for an APD on the right side, which was confirmed though evaluation by

ABBREVIATIONS ACOA = anterior communicating artery; APD = afferent pupillary defect; $C O N$ = compressive optic neuropathy; SAH = subarachnoid hemorrhage; WFNS $=$ World Federation of Neurosurgical Societies.

SUBMITTED October 19, 2016. ACCEPTED February 9, 2017.

INCLUDE WHEN CITING Published online August 25, 2017; DOI: 10.3171/2017.2.JNS162654. 

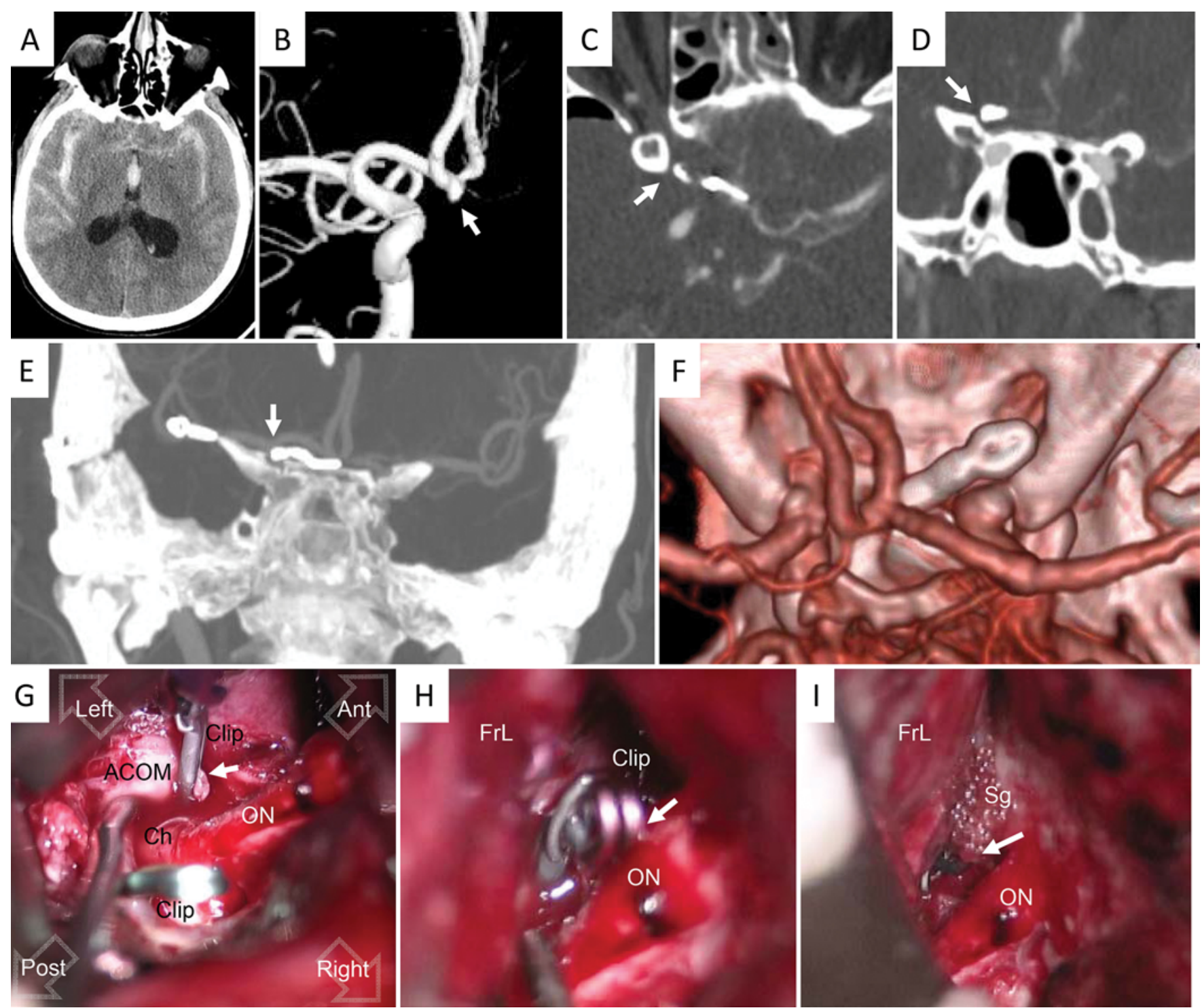

FIG. 1. Case 1. Head CT scan (A) demonstrates diffuse SAH with intraventricular extension secondary to a bilobed ACoA aneurysm with one dome measuring $2.6 \mathrm{~mm}$ in diameter and the second measuring $3.1 \mathrm{~mm}$, with a total height of $5 \mathrm{~mm}$ and a neck measuring $2.6 \mathrm{~mm}$ on 3D rotational cerebral angiogram (arrow) (B). Postoperative CT angiograms show occlusion of the aneurysm by a clip that projects toward the optic canal (arrows) (C-F). Intraoperative microscope images demonstrate the aneurysm clip in proximity to the optic nerve $(\operatorname{arrow})(\mathbf{G})$, which comes to lie in proximity to the nerve when retraction is removed (arrow) $(\mathbf{H})$. The clip is repositioned medially and held in place by Surgicel so that it may rest away from the nerve on the floor of the anterior fossa (arrow) (I). ACOM = ACoA; Ant = anterior; $\mathrm{Ch}=$ chiasm; FrL = frontal lobe; ON = optic nerve; Post = posterior; Sg = Surgicel. Figure is available in color online only.

the ophthalmology team. The remainder of the patient's neurological status was unchanged, and a head CT scan was negative for intracranial hemorrhage. On cranial reexploration, the clip was seen to be compressing the optic nerve when frontal lobe retraction was released. The original clip was removed and a compatible side-biting clip was applied with the left hand so the head of the clip was pointing medially and resting on the tuberculum sella. This was held in place by applying Surgicel (Ethicon) absorbable hemostatic gauze to the head of the clip and attaching it to the anterior cranial base (Video 1).

VIDEO 1. Case 1. Intraoperative microscope footage demonstrates that with frontal lobe retraction the aneurysm clip appears to be sit- uated well away from the optic nerve. However, on release of frontal lobe retraction, the clip comes to rest adjacent to the optic nerve. This persists despite repositioning of the clip. After repositioning and a "sling" of hemostatic cellulose, the clip instead rests medially on the floor of the anterior fossa. Copyright University of Michigan Health System. Published with permission. Click here to view.

Upon discharge and at her 3-month follow-up appointment, the patient was found to have made a complete recovery. Her APD was resolved and she had no residual neurological deficits.

\section{Case 2}

A 72-year-old woman with a history of hypertension 

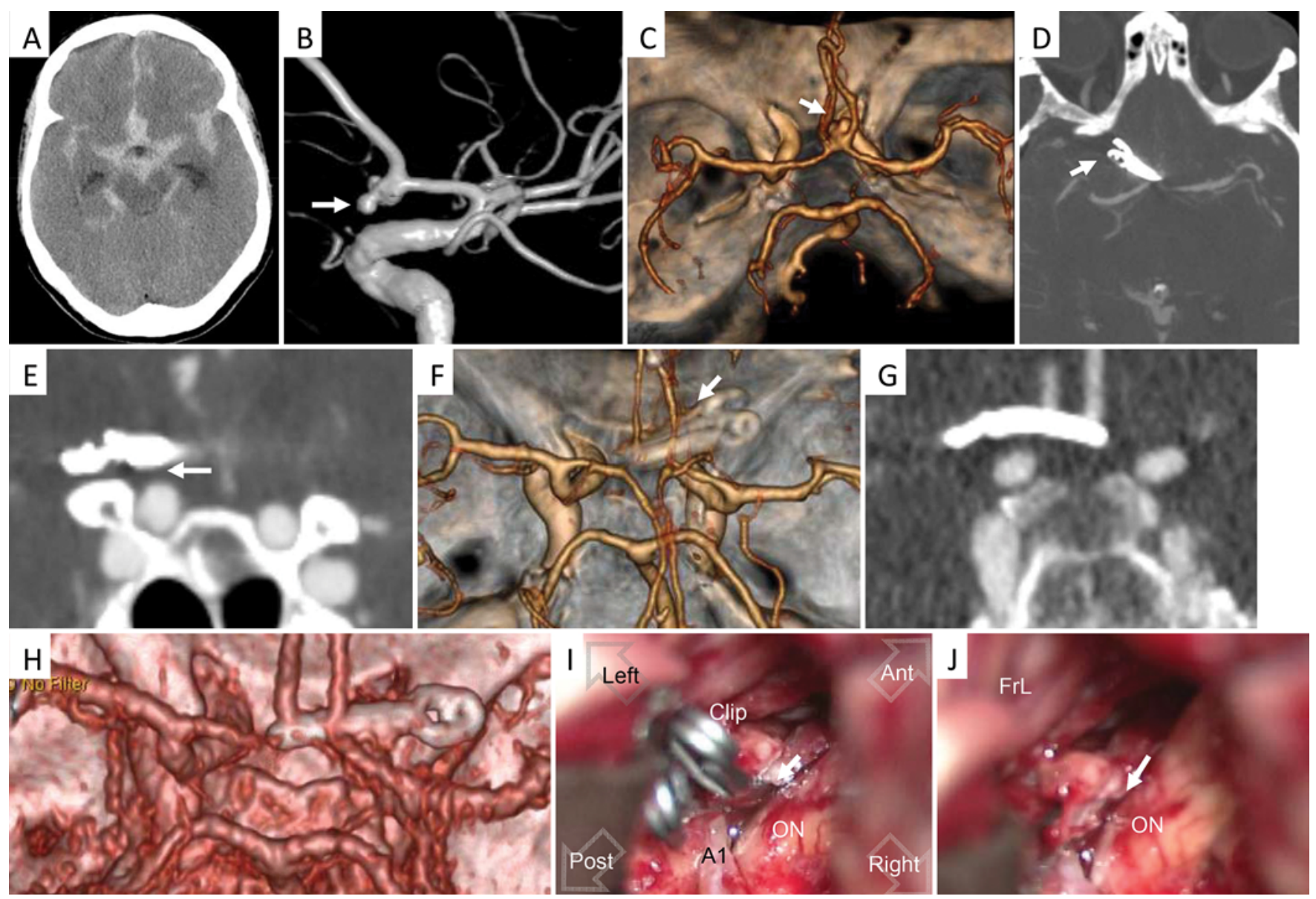

FIG. 2. Case 2. Head CT scan (A) demonstrates diffuse SAH in the basal cisterns and a complex, multilobulated ACoA aneurysm measuring about $6.8 \mathrm{~mm}$ in height and $6.9 \mathrm{~mm}$ in diameter with a $5.3-\mathrm{mm}$ neck, as demonstrated on 3D rotational cerebral angiograms (arrows) (B and C). Initial postoperative CT angiogram reveals 2 clips used for occlusion of aneurysm dome; clips are lying immediately superior to the optic canal (arrows) (D-F). After exploration, one clip was left in place and rests over the anterior clinoid process ( $\mathbf{G}$ and $\mathbf{H})$. Intraoperative microscope image shows the second aneurysm clip in proximity to the optic nerve (arrow) (I). This compression is resolved after removal of the reinforcing clip $($ arrow $)(J)$. Ant = anterior; $A 1=A_{1}$ segment of the anterior cerebral artery; FrL = frontal lobe; $\mathrm{ON}=$ optic nerve; Post = posterior. Figure is available in color online only.

presented with headache and syncope. Head CT revealed modified Fisher Grade 4 SAH (Fig. 2). Clinically the patient's status was characterized as a Hunt and Hess Grade III and WFNS Grade II. A diagnostic angiogram revealed a wide-necked, multilobulated ACoA aneurysm.

Prior to the procedure, a ventriculostomy catheter was placed. A right-sided frontotemporal craniotomy was performed for microsurgical clip ligation. A slightly curved Mizuho MRI-compatible clip was applied across the aneurysm neck, resulting in complete occlusion of the aneurysm. A second identical clip was applied across the top to obliterate the dome of the aneurysm. The heads of both clips were pointed away from the ACoA, toward the right optic nerve.

On postoperative Day 14, the patient developed a rightsided APD during the process of weaning her from the ventriculostomy catheter. After CT revealed hydrocephalus, we decided to pursue ventricular drainage. After 6 hours of drainage, the APD did not resolve and the patient was taken to the operating room for reexploration, where we observed that the second clip, which was on the dome of the aneurysm, appeared to be compressing the optic nerve. The aneurysm was trapped with the patient in burst suppression and the top clip was removed (Video 2) and not replaced, as the bottom clip had obliterated the entirety of the aneurysm.

VIDEO 2. Case 2. Intraoperative microscope footage reveals the reinforcing aneurysm clip to be possibly impinging on the optic nerve. This reinforcing clip is removed, with the remaining aneurysm clip lying on the anterior clinoid process. Copyright University of Michigan Health System. Published with permission. Click here to view.

On discharge, the APD had improved.

\section{Case 3}

A 59-year-old man with a history of hypertension presented with dizziness and tinnitus and was found to have an ACoA aneurysm.

A right frontotemporal craniotomy was performed. A $45^{\circ}$-angle standard Yaşargil clip (Aesculap) was placed across the neck of the aneurysm (Video 3). 

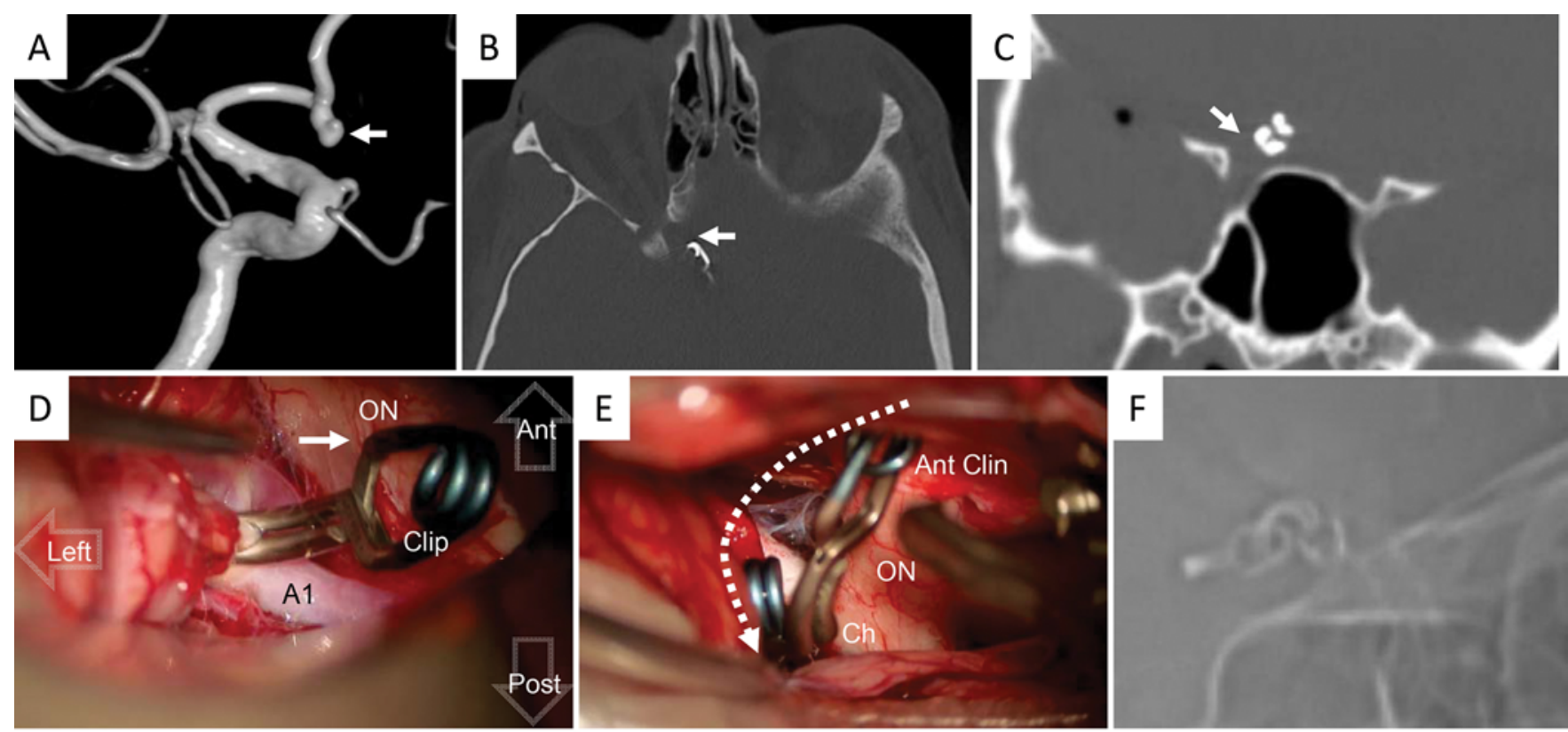

\section{$\mathrm{F}$}
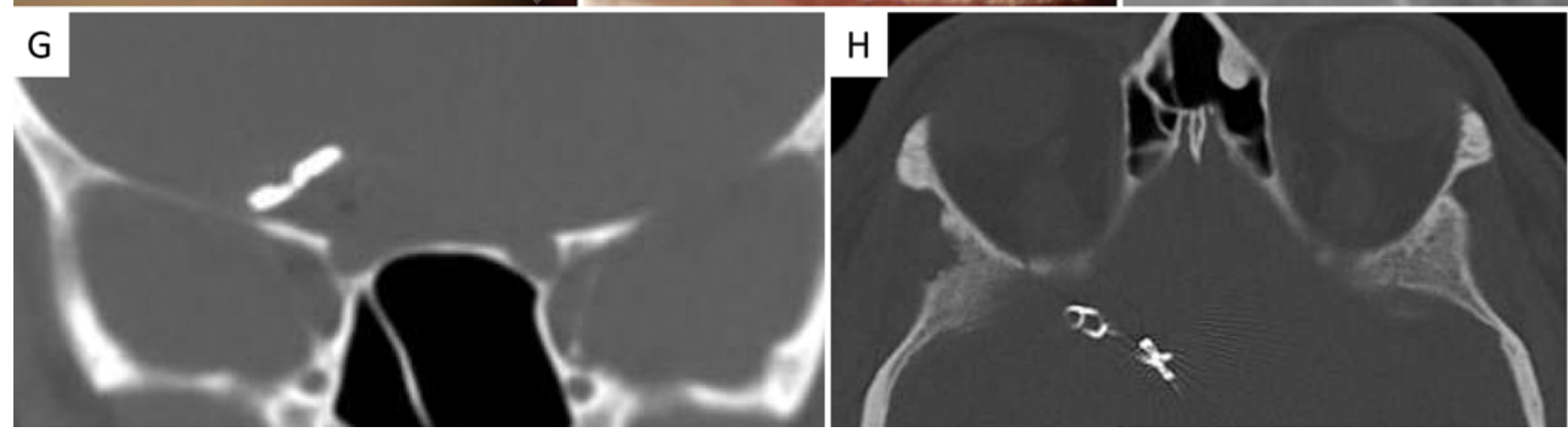

FIG. 3. Case 3. 3D rotational angiogram demonstrating a $5 \times 4-\mathrm{mm}$ ACoA aneurysm with a $3-\mathrm{mm}$ neck (arrow) (A). Immediate postoperative CT scans show the clip positioned close to the optic canal (arrows) (B and C). At the index operation, the aneurysm clip was adjacent to the optic nerve (arrow) (D). The patient awoke with vision loss in the right eye despite our padding the clip with Gelfoam. The patient was returned to the operating room and a clip "bridge" was constructed (dashed arrow) (E), with postoperative CT scans $(\mathrm{F}-\mathrm{H})$ demonstrating adequate distance from the optic canal. Ant = anterior; Ant Clin = anterior clinoid process; $\mathrm{A} 1=$ $\mathrm{A}_{1}$ segment of the anterior cerebral artery; $\mathrm{Ch}=$ chiasm; $\mathrm{ON}=$ optic nerve; Post = posterior. Figure is available in color online only.

VIDEO 3. Case 3. Intraoperative microscope footage demonstrates proximity of the aneurysm clip (with a reinforcing clip) to the optic nerve. A Gelfoam sponge is placed in an effort to displace the aneurysm clips and pad the nerve. After learning that the patient exhibits visual loss and taking him back to the operating room, we remove the original aneurysm clips and a $90^{\circ}$ clip is placed onto the aneurysm neck. This is followed by affixing a side-angled clip to the spring coil of the primary clip, creating a "bridge" to redirecting the clip forces over the optic nerve and onto the anterior clinoid process. The spring coil of the bridging clip is sutured onto the dura over the anterior clinoid process to maintain this arrangement. Copyright University of Michigan Health System. Published with permission. Click here to view.

A second microclip of the same shape was placed across the dome of the aneurysm. A small pledget of Gelfoam (Pfizer) absorbable gelatin sponge was placed between the optic nerve and the clip for safety, although no active compression was observed.

On awakening, the patient noted complete loss of vision in his right eye. CT scanning demonstrated only normal postoperative changes. The patient was taken back to the operating room for reexploration and clip adjustment. The bottom clip was removed and replaced with a $90^{\circ}$-angle clip. The upper clip was also removed. It was not replaced, as the larger $90^{\circ}$-angle clip completely obliterated the aneurysm. A "bridge" was created with a side-angle clip placed onto the spring coil of the $90^{\circ}$-angle clip, redirecting it completely away from the optic nerve (Fig. 3, Video 3).

Following this surgery, the patient indicated that he had improved vision in the right eye. The APD was much improved, although not completely resolved.

\section{Discussion}

CON while treating carotid artery ophthalmic, superior hypophyseal, and ACoA aneurysms is plausible secondary to potential coil-induced expansion of the aneurysm or manipulation of the optic nerve in microdissection. ${ }^{3,5}$, ${ }^{7-10}$ However, we have not found any case reports describing delayed-onset APD secondary to clip movement causing $\mathrm{CON}$, with subsequent clip repositioning leading to 
reversal of injury. We report 3 cases in which CON was detected after clip ligation of ACoA aneurysms and clip repositioning that led to reversal of the CON.

All cases involved clipping of the ACoA aneurysm. In each case, the clip head was superior to the optic nerve. As the retractor is removed, the anterior cerebral artery, $\mathrm{ACoA}$, and clips can be pushed anteriorly, thus compressing the optic nerve. It is imperative to perform a detailed optic nerve examination (gross visual fields and pupillary reactivity) for patients undergoing clip ligation of ACoA aneurysms. Such evaluation is essential for encephalopathic or comatose patients who will not be able to complain of visual dysfunction.

As exemplified by our cases, both recognition of visual dysfunction and early intervention are crucial. At our institution, we now evaluate the potential position of the clip head as the retractor is removed and the patient is placed in the reverse Trendelenburg position to emulate future patient head elevation. If we feel the clip head will press against the optic nerve, we place strips of Surgicel on the clip head and attach the Surgicel to the cerebrum or tuberculum sella to guide the clip head away from the optic nerve. If possible, clipping the aneurysm in a way that places the clip head medial to the optic nerve will prevent any chance of CON. We have also seen that applying Gelfoam to prevent clip compression of the optic nerve may lead to added compression as the Gelfoam swells. Given the proximity of the ACoA complex to the optic nerve, we try to use a single clip to minimize clip bulk and potential CON. We advocate changing the clip position or using materials to change the potential clip head trajectory after closure if compression seems inevitable. While CON may be the cause of optic nerve dysfunction, it is plausible that such injury is the result of vascular insult during an aneurysm dissection. Sequelae following a vascular injury could be immediate or occur in a delayed fashion due to vasospasm or a hypotensive episode. Future studies could investigate the difference in prevalence between postoperative APD secondary to CON compared with vascular injury. With the knowledge that CON can occur immediately postoperatively or in a delayed fashion, visual acuity should be tested in the hospital and at postoperative clinic visits.

Two purposes of this article were to emphasize early recognition of this complication and to suggest several methods to alleviate vision loss. However, this investigation is limited due to lack of formal visual acuity and visual field examinations over the long term. In the future, we suggest that clinicians and researchers obtain quantitative data to precisely measure deficits and subsequent improvements.

\section{Conclusions}

Increased risk of CON may be associated with $\mathrm{ACoA}$ aneurysm clipping. With close surveillance, $\mathrm{CON}$ may be quickly identified and reversed with operative repositioning of microsurgical clips using a variety of techniques.

\section{References}

1. Andaluz N, Van Loveren HR, Keller JT, Zuccarello M: Ana- tomic and clinical study of the orbitopterional approach to anterior communicating artery aneurysms. Neurosurgery 52:1140-1149, 2003

2. Date I, Asari S, Ohmoto T: Cerebral aneurysms causing visual symptoms: their features and surgical outcome. Clin Neurol Neurosurg 100:259-267, 1998

3. de Oliveira JG, Borba LA, Rassi-Neto A, de Moura SM, Sanchez-Júnior SL, Rassi MS, et al: Intracranial aneurysms presenting with mass effect over the anterior optic pathways: neurosurgical management and outcomes. Neurosurg Focus 26(5):E3, 2009

4. Giannotta SL, Oppenheimer JH, Levy ML, Zelman V: Management of intraoperative rupture of aneurysm without hypotension. Neurosurgery 28:531-536, 1991

5. Heran NS, Song JK, Kupersmith MJ, Niimi Y, Namba K, Langer DJ, et al: Large ophthalmic segment aneurysms with anterior optic pathway compression: assessment of anatomical and visual outcomes after endosaccular coil therapy. $\mathbf{J}$ Neurosurg 106:968-975, 2007

6. Iwamoto H, Kiyohara Y, Fujishima M, Kato I, Nakayama $\mathrm{K}$, Sueishi K, et al: Prevalence of intracranial saccular aneurysms in a Japanese community based on a consecutive autopsy series during a 30 -year observation period. The Hisayama study. Stroke 30:1390-1395, 1999

7. La Pira B, Brinjikji W, Hunt C, Chen JJ, Lanzino G: Reversible edema-like changes along the optic tract following pipeline-assisted coiling of a large anterior communicating artery aneurysm. J Neuroophthalmol [epub ahead of print], 2016

8. Liu P, Lv X, Li Y, Lv M: High resolution MRI in treatment decision of anterior communicating artery aneurysm accompanied by visual symptoms: Endovascular treatment or surgical clipping? A report of two cases and literature review. Interv Neuroradiol 22:270-277, 2016

9. Park JH, Park SK, Kim TH, Shin JJ, Shin HS, Hwang YS: Anterior communicating artery aneurysm related to visual symptoms. J Korean Neurosurg Soc 46:232-238, 2009

10. Schmidt GW, Oster SF, Golnik KC, Tumialán LM, Biousse $\mathrm{V}$, Turbin R, et al: Isolated progressive visual loss after coiling of paraclinoid aneurysms. AJNR Am J Neuroradiol 28:1882-1889, 2007

\section{Disclosures}

The authors report no conflict of interest concerning the materials or methods used in this study or the findings specified in this paper.

\section{Author Contributions}

Conception and design: Pandey, Thompson. Acquisition of data: all authors. Analysis and interpretation of data: all authors. Drafting the article: Linzey, Chen, Savastano. Critically revising the article: Pandey, Savastano. Reviewed submitted version of manuscript: all authors. Approved the final version of the manuscript on behalf of all authors: Pandey. Study supervision: Pandey, Thompson.

\section{Supplemental Information \\ Videos}

Video 1. https://vimeo.com/210774325.

Video 2. https://vimeo.com/210774677.

Video 3. https://vimeo.com/210774836.

\section{Correspondence}

Aditya S. Pandey, Department of Neurosurgery, University of Michigan, 1500 E Medical Center Dr., Rm. 3552 TC, Ann Arbor, MI 48109-5338. email: adityap@med.umich.edu. 